\title{
Excisional keratectomy combined with focal cryotherapy and amniotic membrane inlay for recalcitrant filamentary fungal keratitis: A retrospective comparative clinical data analysis
}

\author{
YINGXIN CHEN $^{1}$, MINGHONG GAO ${ }^{1}$, JOSHUA K. DUNCAN ${ }^{2}$, DI RAN ${ }^{3}$, \\ DENISE J. ROE ${ }^{3}$, MICHAEL W. BELIN ${ }^{2}$ and MINGWU WANG ${ }^{2}$
}

\author{
${ }^{1}$ Department of Ophthalmology, The General Hospital of Shenyang Military Command, Shenyang, \\ Liaoning 110016, P.R. China; ${ }^{2}$ Department of Ophthalmology and Visual Science, College of Medicine, \\ The University of Arizona, Tucson, AZ 85711; ${ }^{3}$ Epidemiology and Biostatistics Division, Mel and Enid \\ Zuckerman College of Public Health, The University of Arizona, Tucson, AZ 85724, USA
}

Received August 20, 2015; Accepted July 21, 2016

DOI: $10.3892 / \mathrm{etm} .2016 .3699$

\begin{abstract}
The aim of the present study was to investigate the efficacy of a novel surgical intervention, excisional keratectomy combined with focal cryotherapy and amniotic membrane inlay (EKCAI), for the treatment of recalcitrant filamentary fungal keratitis. A retrospective analysis was performed of patients who underwent excisional keratectomy combined with conjunctival flap inlay (EKCFI), EKCAI or therapeutic penetrating keratoplasty (TPK) from January 2006 to January 2011. Recalcitrance was determined as being unresponsive to standard medical antifungal therapy for at $\geq 1$ week. Outcome measures among the three intervention modalities were compared. A total of 128 patients had a follow-up of $\geq 1$ year after the primary intervention. The success rates of interventions at 1-year follow-up were $58.33 \%$ in the EKCFI group, $88.37 \%$ in the EKCAI group and $93.44 \%$ in the TPK group $(\mathrm{P}<0.0002)$. The preoperative visual acuity of the three groups were similar $(\mathrm{P}=0.6458)$, while the postoperative best-corrected visual acuity (BCVA) of patients without recurrence was significantly different among the three groups 3 months after surgery. The best postoperative BCVA was found in the TPK group, while the worst was in the EKCFI group. In conclusion, EKCAI does not require donor cornea, is straightforward surgically, and has a favorable success rate compared with EKCFI.
\end{abstract}

Correspondence to: Dr Minghong Gao, Department of Ophthalmology, The General Hospital of Shenyang Military Area Command, 83 Wenhua Road, Shenyang, Liaoning 110016, P.R. China

E-mail: gmhgmh00000@163.com

Key words: refractory fungal keratitis, cryotherapy, conjunctival flap, amniotic membrane inlay, therapeutic keratoplasty

\section{Introduction}

Fungal keratitis has an prominently increasing morbidity, accounting for $46.7 \%$ of purulent corneal infection in China (1). It has been reported that filamentary fungi are the most prevalent pathogens in China, wherein the genus Fusarium is the most common cause (77.6\%) followed by Aspergillus (10.8\%) (2). A well-known contributing factor for the development of fungal keratitis is ocular trauma, specifically contamination of corneal lesions by soil and other vegetative material (3). Notably, the majority of the patients suffering from fungal keratitis in China are farmers in whom early diagnosis is easily missed, and whose residences are commonly distant from well-equipped eye care facilities. Delays in diagnosis and the initiation of prompt antifungal medical therapy are the major reasons that many patients from rural areas present with advanced corneal infection (4). Since approximately one-third of cases of fungal keratitis result in either medical treatment failures or corneal perforations (5), it has become a serious disease in China. When fungal corneal infections become unresponsive to medical therapy, surgical intervention offers a second chance for eradicating the infection and maintaining the globe integrity.

Although therapeutic penetrating keratoplasty (TPK) and lamellar keratoplasty (LK) have been shown to be effective in the management of recalcitrant fungal keratitis (6-9), the lack of donor corneas in China has forced ophthalmologists to investigate other treatment strategies. Given the massive rejection reaction and high graft failure rates associated with keratoplasty in the treatment of recalcitrant fungal keratitis, any modality that avoids the requirement for a donor cornea would be of significant value in countries such as China $(1,10,11)$.

Historically, debulking the organism and necrotic material by daily debridement at the slit lamp was the mainstay of therapy for fungal keratitis. The removal of active infection and devitalized tissue was conducted with the aim of enhancing the penetration of topical antifungal medications. However, removal of the necrotic corneal tissue combined with conjunctival flap excisional keratectomy combined with conjunctival 
flap inlay (EKCFI) is now becoming widely used in many tertiary eye care facilities in China (12). Additionally, cryotherapy combined with antifungal agents and/or corneoscleral grafting has been used successfully in cases of fungal scleritis and keratoscleritis $(13,14)$. Several groups have reported promising results using human amniotic membrane as an adjunct for the treatment of active microbial keratitis (15-17). In a study conducted by Chen et al in 2006, human amniotic membrane was successfully used in active cases of fungal keratitis, even in some cases in which perforation had previously occurred (18). These earlier reports demonstrate that non-keratoplasty modalities may be effective alternatives for the treatment of recalcitrant fungal keratitis.

In 2006, the present authors began employing human amniotic membrane and cryotherapy as adjuncts to surgical interventions in the management of fungal keratitis using excisional keratectomy combined with focal cryotherapy and amniotic membrane inlay (EKCAI). The present study is a retrospective analysis of all confirmed cases of filamentary fungal keratitis at a single institution, in which biostatistical analysis was attempted to evaluate the efficacy of EKCAI compared with that of conventional surgical therapies for recalcitrant fungal keratitis.

\section{Materials and methods}

Patient enrolment and ethics. The charts of all patients with a diagnosis of fungal keratitis who were enrolled in the General Hospital of Shenyang Military Command (Shenyang, China) in-patient ophthalmology service from January 2006 to January 2011, were reviewed and the cases that received surgical intervention were retrieved from the records. Inclusion criteria in this analysis were as follows: i) Filamentary keratitis confirmed by corneal scrape culture or potassium hydroxide staining; ii) any cases of urgent surgical intervention due to corneal perforation either at presentation or within the first week of admission; iii) cases deemed as medical treatment failure, defined as documentation of progression of corneal ulcers after at least 1 week of appropriate medical treatment; iv) patients who underwent surgical interventions and had $\geq 1$ year of follow-up after surgery.

Medical treatment was standardized to topical 5\% natamycin eye drops (5\%; Alcon; Novartis International AG, Basel, Switzerland) every $2 \mathrm{~h}$ and $150 \mathrm{mg}$ intravenous infusion of fluconazole (Changchun Dirui Pharmacy, Inc., Changchun, China) once a day from the time of admission. A total of 128 eyes (128 patients) met the inclusion criteria and were selected in this analysis. The study was approved by the ethics committee of the General Hospital of Shenyang Military Command and conducted in accordance with the Declaration of Helsinki. Written informed consent was obtained from each subject.

Surgical triage of fungal keratitis cases. As a general rule, cases with corneal perforation either at presentation or during medical treatment were managed with TPK if donor corneas were available. Otherwise, such patients were treated with EKCFI or EKCAI, based on the availability of amniotic membranes at the time of surgeries or the surgeon's personal preference. If patients were deemed to be a medical treatment failure, cases with corneal ulcers with the depth $>70 \%$ of the corneal stroma were also preferentially treated with TPK if donor corneas were available. Therefore, relatively severe cases in the clinic tended to be treated with TPK. For cases involving perforation as a result of medical treatment failure that did not receive TPK, the assignment of either EKCFI or EKCAI was not randomized either, with more EKCAI procedures performed in later phase of the reviewed time frame.

Surgical procedures and postoperative treatment. All surgical interventions were performed under general or local retrobulbar anesthesia with $2.5 \mathrm{ml} \mathrm{2 \%}$ lidocaine. Intraoperatively, $2 \mathrm{mg} / \mathrm{ml}$ fluconazole solution was used to irrigate the corneal ulcers for subconjunctival injection. The operated eyes were bandaged for $12 \mathrm{~h}$ postoperatively prior to the initiation of treatment. The postoperative medical antifungal regimen was the same in all three treatment groups and consisted of 5\% natamycin drops topically 6 times daily for the first week, 4 times daily for the second week, and twice daily for the following 2 additional weeks during waking hours. In addition, $1 \%$ atropine sulfate ointment (1\%; Alcon; Novartis International AG) was applied once daily.

EKCFI procedure. Details of the EKCFI procedure have been described previously by Sun et al (12). Briefly, the necrotic corneal tissue was removed with sharp dissection and two 5-mm bulbar conjunctival incisions were made perpendicular to the limbus at the 3:00 and 9:00 o'clock positions, respectively. The incisions were extended circumferentially along the limbus to create superior and inferior conjunctival flaps, and then undermined to the fornices. Two conjunctival flaps were then pulled centripetally onto the corneal surface and sutured in an interrupted manner to cover the debrided stromal ulcer bed.

EKCAI procedure. In the EKCFI procedure, after the ulcer bed was debrided and dried with a surgical sponge, the entire area of the ulcer was treated with a 3-mm cryoprobe, with involvement of the junctional region $1 \mathrm{~mm}$ beyond the edge of the ulcer. The duration of each treatment was $10 \mathrm{sec}$ once the probe reached $-70^{\circ} \mathrm{C}$. Dry amniotic membrane (Ruiji Biotechnology Co. Ltd., Jiangxi, China) was first hydrated for $10 \mathrm{~min}$ in balanced salt solution (BSS) solution. Multilayers of the amniotic membrane were placed as inlays to cover the ulcer bed and sutured onto the stroma with interrupted $10-0$ nylon sutures.

TPK procedure. The specific TPK process was reported by Lim et al in 2011 (9). In summary, a trephine that was $\geq 0.5 \mathrm{~mm}$ larger in diameter than the affected area was applied to each case to perform the penetrating keratoplasty. Prior to graft placement, the hypopyon in the anterior chamber was thoroughly irrigated with $2 \mathrm{mg} / \mathrm{ml}$ fluconazole solution and any fibrovascular membrane present on the anterior iris surface was carefully removed. The donor corneal grafts were generally oversized by $0.25-0.5 \mathrm{~mm}$. Large corneal or corneoscleral grafts were required for ulcers with limbal or scleral involvement. Off-center grafts were placed when required to adequately cover the defects.

Outcome evaluation. Therapeutic success, recurrence of the cornea or sclera infection, postoperative best-corrected 
Table I. Patient characteristics in the three groups.

\begin{tabular}{|c|c|c|c|c|}
\hline Index & EKCAI $(n=43)$ & EKCFI $(n=24)$ & TPK $(n=61)$ & $P$-value \\
\hline Male gender & $25(58.14)$ & $21(87.50)$ & $38(62.30)$ & 0.0396 \\
\hline Age (years) & $46.02 \pm 5.51$ & $43.42 \pm 6.03$ & $44.69 \pm 7.18$ & 0.2713 \\
\hline \multicolumn{5}{|l|}{ History } \\
\hline Trauma $^{\mathrm{b}}$ & $19(44.19)$ & $15(62.50)$ & $27(44.26)$ & 0.2713 \\
\hline Surgery ${ }^{b}$ & $1(2.33)$ & $1(4.17)$ & $2(3.28)$ & 1.0000 \\
\hline Steroid use ${ }^{b}$ & $14(32.56)$ & $6(25.00)$ & $2(3.28)$ & $<0.0001$ \\
\hline Contact lens use $\mathrm{b}^{\mathrm{b}}$ & $1(2.33)$ & $1(4.17)$ & $1(1.64)$ & 0.7695 \\
\hline No positive history ${ }^{\mathrm{b}}$ & $4(9.30)$ & $4(16.67)$ & $15(24.59)$ & 0.1507 \\
\hline \multicolumn{5}{|l|}{ Presentation } \\
\hline LogMAR vision & $1.81 \pm 0.20$ & $1.77 \pm 0.20$ & $1.80 \pm 0.20$ & 0.6458 \\
\hline Ulcer diameter, $\mathrm{mm}$ & $6.51 \pm 1.71$ & $6.50 \pm 1.72$ & $6.97 \pm 1.47$ & 0.2720 \\
\hline Ulcer depth (\%) & $69.53 \pm 24.39$ & $71.25 \pm 26.59$ & $73.61 \pm 23.88$ & 0.7028 \\
\hline Infection WLI & $9(20.93)$ & $4(16.67)$ & $15(24.59)$ & 0.7166 \\
\hline Hypopyon present ${ }^{b}$ & $38(88.37)$ & $20(83.33)$ & $55(90.16)$ & 0.6225 \\
\hline Perforation $^{\mathrm{b}}$ & $10(23.26)$ & $5(20.83)$ & $19(31.15)$ & 0.5215 \\
\hline
\end{tabular}

Values are presented as mean \pm standard deviation or number $(\%)$ as appropriate. ${ }^{\text {a }}$-values based on Pearson Chi-square tests for categorical variables and analysis of variance for continuous variables; ${ }^{b} \mathrm{P}$-values based on Fisher's exact tests. EKCAI, excisional keratectomy combined with focal cryotherapy and amniotic membrane inlay; EKCFI, excisional keratectomy combined with conjunctival flap inlay; TPK, therapeutic penetrating keratoplasty; WLI, with limbal involvement.

visual acuity (BCVA), postoperative complications, corneal neovascularization in the EKCFI and EKCAI groups, and graft survival (clarity) in the TPK group, were all reviewed during a 1-year follow-up. Therapeutic success was defined as no evidence of recurrent infection following the cessation of postoperative antifungal medical therapy and the maintenance of globe integrity without subsequent secondary surgical intervention during 1-year follow-up. Therapeutic failure was defined as: i) Necessity to continue medical treatment for $\geq 3$ months following the primary surgery; ii) recurrence of the original infection during the 1-year follow-up period following the cessation of antifungal medical therapy, requiring repeated medical therapy or secondary surgical intervention. Corneal neovascularization was graded on a scale of 0 to IV, based on its involvement of the cornea by quadrants at 1 year postoperatively in cases with therapeutic success. Postoperative complications included corneal perforation, development of endophthalmitis and secondary glaucoma within 1 year of the primary surgeries. The conversion of the original decimal notation visual acuity to LogMAR vision was performed to facilitate statistical analysis. For vision $\mathrm{CF}$ or worse, the following conversions were made: $\mathrm{CF}=1.6$; hand movements $=2.0$; light perception $=2.5$; and no light perception $=3.0$ LogMAR units, according to Arroyo et al (19).

Statistical analysis. The dataset, including patient's demographic characteristics, preoperative findings and postoperative results among the three surgical interventions, was carefully evaluated by expert statisticians. Analysis of variance was used for normally distributed continuous variables to test for mean differences within the surgical groups. Pearson Chi-square test was used to test the frequency differences between groups for categorical variables. However, when categorical variables had a small number of expected counts within at least one of the three surgical categories, the Fisher's exact test was used instead. In addition, pairwise comparisons of preoperative data and postoperative results among the three surgical groups were conducted, with Bonferroni adjustment.

Odds ratios and $95 \%$ confidence intervals were determined by Firth logistic regression, a penalized likelihood method to solve quasi-complete separation problems in regular logistic regression models. Three logistic regression models were used to explore the association between surgical success and surgical interventions. Unadjusted results were derived from the initial univariate model. Model 1 was adjusted for gender. Model 2 was adjusted for both gender and a history of steroid use (positive vs. negative). $\mathrm{P}<0.05$ was considered significant to indicate a statistically significant difference. All analyses were conducted using SAS software, version 9.4 (SAS Institute, Inc., Cary, NC, USA).

\section{Results}

Preoperative characteristics. The preoperative characteristics of all selected patients, included pertinent history, infection characteristics and vision are shown in Table I, There was a male predominance, and histories of steroid use were not evenly distributed among the three intervention groups. There were predominantly more males in the EKCFI group and more cases of steroid use in the EKCAI group, in comparison with the other two groups respectively (Table II). No statistical differences were found among the groups in terms of disease characteristics and severity. All patients who met the selection criteria had vision defined as count fingers (CF) or worse at presentation. 
Table II. Postoperative outcome distributions among surgical intervention groups.

\begin{tabular}{lccr}
\hline Surgical intervention & EKCAI $(\mathrm{n}=43)$ & EKCFI $(\mathrm{n}=24)$ & TPK (n=61) \\
\hline Therapeutic success $^{\mathrm{a}}$ & $38(88.37)$ & $14(58.33)$ & $57(93.44)$ \\
Secondary glaucoma $^{\mathrm{b}}$ & $5(11.63)$ & $6(25.00)$ & $3(4.92)$ \\
Neovascularization $^{\mathrm{b}}$ & & & 0.0002 \\
Grade 0 & $14(32.56)$ & $7(29.16)$ & $18(29.50)$ \\
Grade I & $10(23.26)$ & $0(0.00)$ & $35(57.38)$ \\
Grade II & $13(30.23)$ & $1(4.17)$ & $7(11.48)$ \\
Grade III & $6(13.95)$ & $15(62.50)$ & $1(1.64)$ \\
Grade IV & $0(0.00)$ & $1(4.17)$ & 0.0001 \\
LogMARvision $^{\mathrm{c}}$ & $1.33 \pm 1.03$ & $1.85 \pm 1.01$ & $0.84 \pm 0.73$
\end{tabular}

Values are presented as mean \pm standard deviation or number $(\%)$ as appropriate. EKCAI, excisional keratectomy combined with focal cryotherapy and amniotic membrane inlay; EKCFI, excisional keratectomy combined with conjunctival flap inlay; TPK, therapeutic penetrating keratoplasty. ${ }^{\mathrm{a} P}$-values based on Chi-square tests; ${ }^{\mathrm{b}} \mathrm{P}$-values based on Fisher's exact tests; ${ }^{\mathrm{C}} \mathrm{P}$-values based on analysis of variance.

Table III. Pairwise comparison of postoperative results.

\begin{tabular}{lccc}
\hline & \multicolumn{3}{c}{ P-value } \\
\cline { 2 - 4 } & $\begin{array}{c}\text { EKCAI vs. } \\
\text { EKCFI }\end{array}$ & $\begin{array}{c}\text { EKCAI vs. } \\
\text { TPK }\end{array}$ & $\begin{array}{c}\text { EKCFI vs. } \\
\text { TPK }\end{array}$ \\
\hline Treatment outcome & 0.0141 & 1 & $<0.0001$ \\
Therapeutic success $^{\mathrm{a}}$ & 0.5472 & 0.8109 & 0.0402 \\
Secondary glaucoma $^{\mathrm{b}}$ & 0.0001 & $<0.0001$ & $<0.0001$ \\
Neuvascularization $^{\mathrm{b}}$ & $<0.1440$ & 0.0171 & $<0.0001$ \\
LogMAR vision $^{\mathrm{c}}$ & 0 & & \\
\hline
\end{tabular}

EKCAI, excisional keratectomy combined with focal cryotherapy and amniotic membrane inlay; EKCFI, excisional keratectomy combined with conjunctival flap inlay; TPK, therapeutic penetrating keratoplasty. ${ }^{\text {P }}$-values based on Pearson Chi-square test; ${ }^{b} \mathrm{P}$-values based on Fisher's exact test; ' $\mathrm{P}$-values based on analysis of variance.

Treatment outcomes. The treatment outcomes of the three interventions are summarized in Table II. TPK achieved the highest rate of infection control (therapeutic success, 93.44\%) compared with EKCAI (88.37\%) and EKCFI (58.33\%). TPK also had the lowest incidence of secondary glaucoma, the least neovascularization of the cornea, and better postoperative vision at 1 year postoperatively, compared with EKCAI and EKCFI. A pairwise comparison was then performed between surgical intervention groups (Table III). Significant differences were observed between the EKCAI and EKCFI groups in infection control $(\mathrm{P}=0.0141)$ and postoperative neovascularization $(\mathrm{P}<0.0001)$. However, there was no significant difference between these two interventions in rates of secondary glaucoma and postoperative vision at 1 year. Compared with EKCAI, TPK showed superior postoperative vision at 1 year $(\mathrm{P}=0.0171)$ and significantly less corneal neovascularization $(\mathrm{P}<0.0001)$. However, no difference was identified in infection control and secondary glaucoma between TPK and EKCAI. TPK also performed significantly better than EKCFI in all outcome measures analyzed $(\mathrm{P}<0.0001)$, with the exception of the rate of secondary glaucoma $(\mathrm{P}=0.0402)$. Typical cases of filamentary fungal keratitis cases treated with different surgical modalities were shown in Fig. 1.

Since the study was not randomized, odds ratios of therapeutic success among surgical interventions were calculated, adjusting for gender and steroid use (Table IV). Univariate modeling shows that EKCAI had superior infection control compared with EKCFI $(\mathrm{P}=0.0073)$, but had an equivalent outcome compared with TPK $(\mathrm{P}=0.3712)$. After adjusting for gender (model 1), gender and steroid use (model 2), the statistical significance remained unchanged.

\section{Discussion}

Filamentous fungal keratitis has a high incidence in China where farmers constitute the majority of the affected patients, and have poor awareness of the signs and symptoms of the disease. In addition, limited availability of services in primary hospitals accounts for the majority of advanced and refractory cases in this region (20). Broad-spectrum antifungal medication is the acknowledged first-line treatment during the earliest stage of the infection. However, a delay in presentation and treatment results in a worse prognosis (21). Analysis of treatment outcomes in cases where treatment had been delayed for 10 days showed significantly higher rates of surgical interventions. In the patients included in the present study, the duration of infection prior to presentation in the majority of cases was $>10$ days, and thus the infection was refractory to antifungal therapy, and required surgical intervention.

Standard surgical modalities, including TPK and LK, have been shown to be effective in the management of recalcitrant fungal keratitis (6-9). However, the low availability of donor corneas has remained a formidable challenge to ophthalmologists in China. Alternative methods are constantly sought for managing these difficult cases. In recent years, EKCFI has been widely adopted in many tertiary eye care facilities in China (12).

In the treatment of serious fungal keratitis, EKCFI is a useful surgical technique, particularly in eye care facilities without keratoplasty capabilities, or when corneal donor tissue is unavailable. It has been theorized that the conjunc- 
Table IV. Odds ratios of therapeutic success among surgical interventions.

\begin{tabular}{|c|c|c|c|c|c|c|c|c|c|}
\hline \multirow{2}{*}{$\begin{array}{l}\text { Surgical } \\
\text { intervention }\end{array}$} & \multicolumn{3}{|c|}{ Univariate model } & \multicolumn{3}{|c|}{ Model $1^{\mathrm{a}}$} & \multicolumn{3}{|c|}{ Model $2^{b}$} \\
\hline & OR & $95 \% \mathrm{CI}$ & P-value & OR & $95 \%$ CI & P-value & OR & $95 \%$ CI & P-value \\
\hline EKCAI & 1 (Ref) & & & 1 (Ref) & & & 1 (Ref) & & \\
\hline EKCFI & 0.184 & $0.054-0.634$ & 0.0073 & 0.087 & $0.019-0.404$ & 0.0018 & 0.077 & $0.016-0.378$ & 0.0016 \\
\hline TPK & 1.874 & $0.473-7.431$ & 0.3712 & 1.806 & $0.440-7.420$ & 0.4121 & 1.451 & $0.339-6.219$ & 0.6161 \\
\hline
\end{tabular}

OR, odds ratio; CI, confidence interval; EKCAI, excisional keratectomy combined with focal cryotherapy and amniotic membrane inlay;

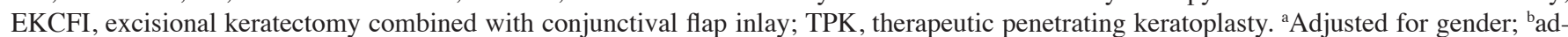
justed for both gender and steroid use.
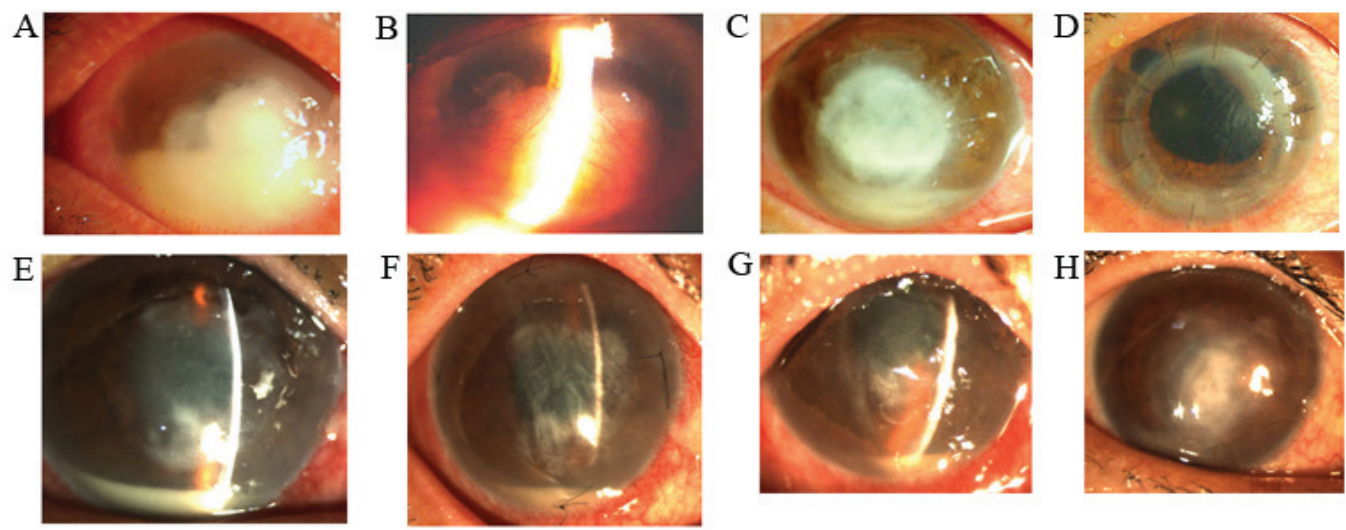

Figure 1. Preoperative and postoperative photographs of filamentary fungal keratitis cases treated with different surgical modalities. (A) A case of central ulcer with $40 \%$ hypopyon and 50\% corneal stromal depth involvement prior to surgery. (B) Three months after treatment with excisional keratectomy combined with conjunctival flap inlay, the hypopyon subsided and the cornea had no signs of infection following the cessation of antifungal medical therapy. (C) A case of large central ulcer with 10\% hypopyon and 70\% corneal stromal depth involvement prior to surgery. (D) Three months after treatment with therapeutic keratoplasty, the transplanted graft remained clear. (E) A case of large central ulcer with $10 \%$ hypopyon and $30 \%$ corneal stromal depth involvement prior to surgery, and at (F) 1 week, $(\mathrm{G}) 1$ month and $(\mathrm{H}) 3$ months after treatment with excisional keratectomy combined with focal cryotherapy and amniotic membrane inlay, respectively.

tival flap provides additional blood supply to the affected area, allowing anti-inflammatory and immunomodulatory substances to be delivered locally, promoting better wound healing. Sun et al (12) reported a total of 10 cases treated with EKCFI, which had a success rate of $100 \%$ with no recurrence. Outcomes of the present study showed that EKCFI had a lower success rate of $58.33 \%(14 / 24)$ compared with that in this earlier study. The severity of disease observed in the present study, combined with the late presentations, and ineffectiveness of antifungal medications may account for the difference in success rates.

EKCAI is a relatively new surgical approach that may be an excellent therapeutic option in the management of refractory fungal keratitis. With outcomes on par with standard TKP in controlling infection and maintaining globe integrity, this technique offers the combined benefits of cryotherapy and amniotic membrane tissue, and does not require donor corneas. Cryotherapy has been used for many years in the treatment of numerous eye diseases with increasing popularity, not only in the United States but also in some developing countries such as China, in the management of infectious keratitis. The therapeutic effects observed with cryotherapy are considered to have several mechanisms, and may be particularly useful in difficult cases of fungal keratitis. Through the destruction of small-caliber blood vessels, tissue ischemia begins to develop. As the freezing continues, osmotic forces produce cellular dehydration which facilitates intracellular ice crystal formation. The ice crystals and intracellular dehydration lead to $\mathrm{pH}$ changes and the formation of a toxic concentration of salts in the cell. Slow thawing produces a longer exposure to this toxic substrate concentration and leads to re-crystallization which increases cell destruction. The ultimate mechanism underlying the effects of cryotherapy is the denaturation of lipid-protein complexes, cytomembrane rupture and eventually cell death (22-24). Cryotherapy has been used in the past to treat acanthamoeba keratitis with variable success, however, its impact in fungal ulcers is not clearly understood (25-28). Early studies have shown promising results in cases of fungal scleritis and keratoscleritis $(12,13)$, and the present study lends further support to its role in these difficult cases. The present authors have previously studied rabbit models with fungal keratitis, which showed the topical cryotherapy is able to destroy fungal elements, and the effect of topical cryotherapy combined with antifungal drugs was observed to be better than that of antifungal drugs alone (29). 
The biological properties attributed to amniotic membrane tissues include lack of immunogenicity, promotion of epithelialization, and inhibition of fibrosis, angiogenesis and inflammation (30-35). The amniotic membrane is the innermost layer of the fetal membrane, and is composed of an avascular epithelial layer over a thick basement membrane. Use of amniotic membrane has been shown to promote epithelialization and reduce inflammation and scarring, possibly through the regulation of growth factors or by acting as a barrier to infiltrating lymphocytes (36). Bauer et al (36) reported that the mechanism underlying the effects of amniotic membrane is associated with the modulation of macrophages. Apoptotic cells induced in the environment of an amniotic membrane support the presence and survival of such macrophages (36).

The use of amniotic membrane in ophthalmology has been extensively reported $(18,37,38)$. The inhibitory effect on inflammation, proteolysis, angiogenesis and fibrosis, and the promoting effect on epithelialization following amniotic membrane transplantation have been well recognized, as well as the potential advantages over traditional penetrating keratoplasty in terms of graft rejection. The application of amniotic membranes for infectious keratitis was reported by Kim et al in early 2001 (39). Unlike TPK or LK, there is no risk of rejection following amniotic membrane transplantation. If the perforation is tiny and, notably, if there is some residual stroma around the perforation site, amniotic membrane transplantation, particularly multilayered, performed at the residual stroma is possible to prevent further leakage of aqueous humor.

With regard to EKCAI, amniotic membrane is extremely valuable for the management of fungal keratitis associated with poor wound healing and impending perforation. Double amniotic membrane and an additional layer of fibrin sealant patch (TachoSil ${ }^{\circledR}$; Baxter, Deerfield, IL, USA) has been reported to be useful for the therapy of large perforations that require urgent treatment (40). Several case reports have also employed the amniotic membrane to cover the ocular surface in the acute stages (36,39-41). This method is aimed at preventing the cicatricial conjunctival and corneal complications that frequently occur in these patients (41).

EKCAI, taking the advantage of the combined benefits of cryotherapy and amniotic membrane, is an effective therapeutic option for curing recalcitrant fungal keratitis. When compared with the traditional method TPK in the present study, there was similar rate of infection control, $93.44 \%$ with TPK compared to $88.37 \%$ in EKCAI. This appeared to be better than EKCFI which had a therapeutic success rate in this study of $58.33 \%$. Additionally, although TPK resulted in a superior postoperative vision at 1 year, and less corneal neovascularization compared with EKCAI, there was no significant difference in the rates of secondary glaucoma. In summary, EKCAI with equivalent therapeutic success, should be considered a valuable alternative in the management of recalcitrant fungal keratitis, particularly in areas where donor corneal tissue is limited.

The major limitation of the present study is the non-randomization of the treatment groups. Due to the limited availability of donor corneas and even amniotic membranes at the time of surgeries, conducting a prospective, randomized study would be problematic. Additionally, corneal ulcers with depth $>70 \%$ were preferentially treated with TPK, if donor corneas were available. Therefore, these patients already had a relatively severe condition. To overcome this possible bias, professional statisticians were invited to this study for the analysis of the data. First, a fairly even distribution of cases was noted in many of the pre-operative characteristics. Furthermore, analysis was also conducted with unevenly distributed parameters being adjusted, such as gender and steroid use, as a cause of infection (Table IV). It appears that the final outcomes were unaffected, with or without the adjustment.

In conclusion, this retrospective study showed that TPK is the first choice for fungal keratitis with late presentation refractory to antifungal drugs, while EKCAI is a better alternative in progressive cases when donor corneas are not available. Such cases have poor prognosis, highlighting the fact that early diagnosis and administration of antifungal treatment is imperative.

\section{References}

1. Xie L, Dong X and Shi W: Treatment of fungal keratitis by penetrating keratoplasty. Br J Ophthalmol 85: 1070-1074, 2001.

2. Xie L, Zhai H, Zhao J, Sun S, Shi W and Dong X: Antifungal susceptibility for common pathogens of fungal keratitis in Shandong Province, China. Am J Ophthalmol 146: 260-265, 2008.

3. Wong TY, Ng TP, Fong KS and Tan DT: Risk factors and clinical outcomes between fimgal and bacterial keratitis: A comparative study. CLAO J 23: 275-281, 1997.

4. Shi WY and Wang T: Several problems of diagnosis and treatment in fungal keratitis in China. Zhonghua Yan Ke Za Zhi 49: 2-5, 2013 (In Chinese).

5. Xie L, Zhai H and Shi W: Penetrating keratoplasty for corneal perforations in fungal keratitis. Cornea 26: 158-162, 2007.

6. Anshu A, Parthasarathy A, Mehta JS, Htoon HM and Tan DT: Outcomes of therapeutic deep lamellar keratoplasty and penetrating keratoplasty for advanced infectious keratitis: A comparative study. Ophthalmology 116: 615-623, 2009.

7. Mandell KJ and Colby KA: Penetrating keratoplasty for invasive fungal keratitis resulting from a thorn injury involving Phomopsis species. Cornea 28: 1167-1169, 2009.

8. Xie L, Qi F, Gao H, Wang T, Shi W and Zhao J: Major shifts in corneal transplantation procedures in north China: 5316 eyes over 12 years. Br J Ophthalmol 93: 1291-1295, 2009.

9. Lim LS, Arundhati A and Tan DT: Sequential therapeutic penetrating keratoplasty with cryopreserved and fresh corneal tissue for severe infectious keratitis: A case-control study. Cornea 30: 739-743, 2011.

10. Xie L, Zhai H and Shi W: Penetrating keratoplasty for corneal perforations in fungal keratitis. Cornea 26: 158-162, 2007.

11. Xie L, Hu J and Shi W: Treatment failure after lamellar keratoplasty for fungal keratitis. Ophthalmology 115: 33-36, 2008.

12. Sun GH, Li SX, Gao H, Zhang WB, Zhang MA and Shi WY: Clinical observation of removal of the necrotic corneal tissue combined with conjunctival flap covering surgery under the guidance of the AS-OCT in treatment of fungal keratitis. Int J Ophthalmol 5: 88-91, 2012.

13. Reynolds MG and Alfonso E: Treatment of infectious scleritis and keratoscleritis. Am J Ophthalmol 112: 543-547, 1991.

14. Rodriguez-AresMT,DeRojas SilvaMV,PereiroM,FenteSampayoB, Gallegos Chamas G and S-Salorio M: Aspergillus fumigatus scleritis. Acta Ophthalmol Scand 73: 467-469, 1995.

15. Heiligenhaus A, Li H, Hernandez Galindo EE, Koch JM, Steuhl KP and Meller D: Management of acute ulcerative and necrotising herpes simplex and zoster keratitis with amniotic membrane transplantation. Br J Ophthalmol 87: 1215-1219, 2003.

16. Al-Kharashi S, Al-Khawaja A, Gonnah el-S, Al-Assiri A Al-Motowa S, Al-Towerki AE and Wagoner MD: Microbial keratitis after amniotic membrane transplantation. Int Ophthalmol 26: 73-76, 2005.

17. Chen JH, Ma DH and Tsai RJ: Amniotic membrane transplantation for pseudomonal keratitis with impending perforation. Chang Gung Med J 25: 144-152, 2002. 
18. Chen HC, Tan HY, Hsiao CH, Huang SC, Lin KK and Ma DH: Amniotic membrane transplantation for persistent corneal ulcers and perforations in acute fungal keratitis. Cornea 25: 564-572, 2006.

19. Arroyo JG, Postel EA, Stone T, McCuen BW and Egan KM: A matched study of primary scleral buckle placement during repair of posterior segment open globe injuries. Br J Ophthalmol 87: $75-78,2003$.

20. Xie L, Zhong W, Shi W and Sun S: Spectrum of fungal keratitis in north China. Ophthalmology 113: 1943-1948, 2006.

21. Rautaraya B, Sharma S, Kar S, Das S and Sahu SK: Diagnosis and treatment outcome of mycotic keratitis at tertiary eye care center in eastern India. BMC Ophthalmol 11: 39, 2011.

22. Fraunfelder FW: Liquid nitrogen cryotherapy for surface eye disease (an AOS thesis). Trans Am Ophthalmol Soc 106: 301-324, 2008.

23. Sullivan JH: Cryosurgery in ophthalmic practice. Ophthalmic Surg 10: 37-41, 1979.

24. Wilkes TD and Fraunfelder FT: Principles of cryosurgery. Ophthalmic Surg 10: 21-30, 1979.

25. Ebrahimi KB, Green WR, Grebe R and Jun AS: Acanthamoeba sclerokeratitis. Graefes Arch Clin Exp Ophthalmol 247: 283-286, 2009.

26. Meisler DM, Ludwig IH, Rutherford I, Bican FE, Langston RH and Visvesvara GS: Susceptibility of acanthamoeba to cryotherapeutic method. Arch Ophthalmol 104: 130-131, 1986.

27. Matoba AY, Pare PD, Le TD and Osato MS: The effects of freezing and antibiotics on the viability of Acanthamoeba cysts. Arch Ophthalmol 107: 439-440, 1989.

28. Binder PS: Cryotherapy for medically unresponsive acanthamoeba keratitis. Cornea 8: 106-114, 1989.

29. Chen Y, Yang W, Gao M, Belin MW, Yu H and $\mathrm{Yu} \mathrm{J}$ Experimental study on cryotherapy for fungal corneal ulcer. BMC Ophthalmol 15: 29, 2015

30. Woo HM, Kim MS, Kweon OK, Kim DY, Nam TC and Kim JH: Effects of amniotic membrane on epithelial wound healing and stromal remodelling after excimer laser keratectomy in rabbit cornea. Br J Ophthalmol 85: 345-349, 2001.
31. Dua HS and Azuara-Blanco A: Amniotic membrane transplantation. Br J Ophthalmol 83: 748-752, 1999.

32. Kubo M, Sonoda Y, Muramatsu R and Usui M: Immunogenicity of human amniotic membrane in experimental xenotransplantation. Invest Ophthalmol Vis Sci 42: 1539-1546, 2001.

33. Akle CA, Adinolfi M, Welsh KI, Leibowitz S and McColl I: Immunogenicity of human amniotic epithelial cells after transplantation into volunteers. Lancet 2: 1003-1005, 1981.

34. Tseng SC, Li DQ and Ma X: Suppression of transforming growth factor-beta isoforms, TGF-beta receptor type II, and myofibroblast differentiation in cultured human corneal and limbal fibroblasts by amniotic membrane matrix. J Cell Physiol 179: 325-335, 1999.

35. Meller D, Pires RT and Tseng SC: Ex vivo preservation and expansion of human limbal epithelial stem cells on amniotic membrane cultures. Br J Ophthalmol 86: 463-471, 2002.

36. Bauer D, Hennig M, Wasmuth S, Baehler H, Busch M, Steuhl KP, Thanos S and Heiligenhaus A: Amniotic membrane induces peroxisome proliferator-activated receptor- $\gamma$ positive alternatively activated macrophages. Invest Ophthalmol Vis Sci 53: 799-810, 2012.

37. Dua HS, Gomes JA, King AJ and Maharajan VS: The amniotic membrane in ophthalmology. Surv Ophthalmol 49: 51-77, 2004.

38. Uhlig CE, Frings C, Rohloff N, Harmsen-Aasman C, Schmitz R, Kiesel L, Eter N, Busse H and Alex AF: Long-term efficacy of glycerine-processed amniotic membrane transplantation in patients with corneal ulcer. Acta Ophthalmol 93: e481-e487, 2015.

39. Kim JS, Kim JC, Hahn TW and Park WC: Amniotic membrane transplantation in infectious corneal ulcer. Cornea 20: 720-726, 2001.

40. Grau AE and Durán JA: Theatment of a large corneal perforation with a multilayer of amniotic membrane and Tachosil. Cornea 31: 98-100, 2012

41. Hsu M, Jayaram A, Verner R, Lin A and Bouchard C: Indications and outcomes of amniotic membrane transplantation in the management of acute Stevens-Johnson Syndrome and toxic epidermal necrolysis: A case-control stydy. Cornea 31: 1394-1402, 2012 